\title{
Mapping of Elastic Behaviour and Tensile Properties of Pressure Therapy Gloves
}

\author{
Nurul Husna Zolkifflee ${ }^{1}$, Siti Hana Nasir ${ }^{1}$, Muhammad Khalis Mohd Idris ${ }^{1}$, Olga Troynikov ${ }^{2}$ \\ ${ }^{1}$ Faculty of Engineering Technology, Universiti Tun Hussein Onn Malaysia, Pagoh Campus, Edu Hub Pagoh, \\ KM1, Jalan Panchor, 84600 Pagoh, Johor, Malaysia, husnawork@gmail.com, sitihana@uthm.edu.my, \\ mkhalis95@gmail.com \\ ${ }^{2}$ School of Engineering, College of Science, Engineering and Health, RMIT University, 3083 Victoria, Australia, \\ olga.troynikov@ rmit.edu.au
}

\begin{abstract}
Pressure therapy gloves is widely practiced by occupational therapists as a treatment for patients with hand arthritis. The gloves are commonly made from elastic materials and constructed to have a negative fit where the size of the unworn glove is smaller than the actual anthropometric measurement. Different levels of elasticity and tensile strength provide varying degrees of fabric tension, thus inducing different degrees of pressure on the users. Restriction of hand movement when wearing a glove will generate high interface pressure between the glove and the hand, which may, apart from discomfort, lead to abrasion and bruising of underlying tissue, especially if the glove fits tightly and its materials are of low elasticity and bending modulus. This study aims to understand the elastic behaviour and tensile properties of pressure therapy gloves during wear and create a hand mapping of its distribution and its relation to fabric stretch. The results showed that the glove stretches up to $56.14 \%$ with the change of postures. The glove stretch maps showed the maximum ranges of fabric stretch located around the metacarpal region. The results of this study will be useful for engineering pressure therapy glove that is comfortable and functional.
\end{abstract}

Key words: Arthritis, elastic behaviour, mapping distribution, therapy gloves

\section{INTRODUCTION}

Arthritis refers to a joint disorder that involves inflammation of one or more joints. There are more than 100 different types of arthritis with different causes and treatment methods, but the two most common types are osteoarthritis (OA) and rheumatoid arthritis (RA). Both OA and RA commonly affect the joints of the hand [1]. The symptoms of arthritis usually develop over time, but it may also appear suddenly. Arthritis is most commonly seen in adults over the age of 65 years old, but it can also develop in children, teens, and younger adults. Gender is also a well-recognized risk factor for arthritis especially in osteoarthritis, with the incidence of hand osteoarthritis is higher in women than men [2]. Joint pain, stiffness and swelling are the most common symptoms of arthritis.
Medical garments are apparel designed for people with medical conditions. These garments can be divided into three main functional categories: protective, treatment and caring. Pressure therapy gloves are garments that belong in the treatment function domain, along with pressure garments, compression stockings and wet dressings [3]. Ready-to-wear gloves are available from several commercial companies, and people with hand OA or RA typically buy these gloves based on clinical recommendations from therapists, consumer websites. Pressure therapy gloves are worn day and/or night to relieve hand symptoms and improve hand function.

Pressure therapy gloves are mostly made of elastic materials and constructed to have a negative fit, where the size of the garment is smaller than that of the body. The patterns of the gloves are normally constructed with $10 \%-20 \%$ reduction (negative fit) in hand circumference in accordance with the fabric's extensibility [4], [5]. The correct proportion and weight of the elastane in the fabric determines its potential tension and allow them to fit better to the hand of the wearer [6], [7]. It allows for better comfort and functionality which ultimately contributes to the wearer's adherence to the intended therapy and the effectiveness of the glove. Inadequate fit causes discomforts and irritations to the skin of the wearer, thereby discouraging their use. In addition, if the glove does not apply the appropriate interface pressure to the underlying tissue, for the intended therapy, it can potentially bring no desired effects or negative effects to the wearer.

The mechanical properties, particularly strength and elongation, are the most important performance properties of knitted fabrics which governs the fabric performance in use by causing a change of dimensions of strained in knitted fabrics [8]. The tensile properties of knitted fabrics have a prominent effect on fabric performance during wear and many other end uses. In many situations, it is important to understand how much the knitted fabric will deform in different directions. The process of deformation of knitted fabrics are defined in the concept of extensibility of knitted fabric and the deformation can be determined and influenced by different factors [9]. As shown by previous studies, the investigation of the elastic behaviour of pressure garments during practical wear is useful for the designing and engineering of functional garments that exert pressure to the underlying body [6], [7], [9]-[11].

In recent years, a new trend in sport and performance garment design and engineering has been proposed that employs the body mapping concept [10], [11]. For example, 
sportswear can be designed based on the mapping of local sweating and heat production patterns, as illustrated in Smith and Havenith [12] and Troynikov and Watson [11]. Material with varying properties, fabric structures and fibre combinations are placed strategically around the body to address the different functional and physiological demands of different body zones. This study aims to understand the elastic behaviour of pressure therapy gloves during wear and create mapping distribution across the hand and to determine the tensile properties of the fabric and its relation to fabric stretch.

\section{MATERIALS AND METHODS}

Two commercially available pressure therapy gloves were selected and tested in this research. These gloves were identified based on previous studies [13] on the current trends in commercial pressure therapy gloves. Both gloves are used to reduce swelling and pain by applying a certain degree of compression, as claimed by the manufacturers. Details of the fabric samples' construction and composition are shown in Table 1. The fibre content information was sourced directly from the manufacturers and/or from the product packaging.

Based on the measurement guide provided by the manufacturers of the gloves and the measurements of the flexible silicone manikin hand, glove size $\mathrm{S}$ was selected for this study for both gloves.

Table 1: Fabric samples' construction and compositions

\begin{tabular}{|c|c|c|}
\hline $\begin{array}{c}\text { Fabric } \\
\text { code }\end{array}$ & Fabric construction & Fibre content (\%) \\
\hline G1 & $\begin{array}{c}\text { Weft-knitted, single } \\
\text { jersey }\end{array}$ & $95 \%$ cotton, 5\% elastane \\
\hline G2 & Warp-knitted, sharkskin & $\begin{array}{c}80 \% \text { polyamide, 20\% } \\
\text { elastane }\end{array}$ \\
\hline
\end{tabular}

\subsection{Structural and physical attributes of the glove}

The gloves were characterized in terms of structural and physical parameters such as mass per unit area, thickness and stitch density. Table 2 shows the test and the standard method used.

Table 2: Structural and physical attributes of the glove

\begin{tabular}{|c|c|}
\hline $\begin{array}{c}\text { Characteristics and } \\
\text { attributes }\end{array}$ & Standard Method \\
\hline Mass Per Unit area & AS2001.2.15- 1987 (1987) (AS, \\
& 2001) \\
\hline Thickness & AS2001.2.15- 1989 (1989) (AS, \\
& 2001) \\
\hline Stitch Density & AS2001.2.6- 2001 (2001) (AS \\
(course and wale) & $2001)$ \\
\hline
\end{tabular}

For fabric mass per unit area, five specimens of $50 \mathrm{~mm} \mathrm{x}$ $50 \mathrm{~mm}$ were cut and weighed, and the mean mass per unit area was calculated. Thickness was measured as a distance between the reference plate and the parallel presser foot of the measuring instrument. Finally, stitch density was measured by counting the number of wales and courses per unit length. Five measurements were taken for both thickness and stitch density and the average reading were recorded.

\subsection{Tensile properties}

ASTM D4964-96 were used to measure the tensile properties of the experimental fabrics. Due to the small amount of fabric able to be cut from the commercial gloves, the sample size for these fabrics was reduced to $50 \mathrm{~mm} \times 50 \mathrm{~mm}$. Three samples in wales and three samples from course were cut from each glove. The test was carried out using Universal Testing Machine. The gauge length was set at $40 \mathrm{~mm}$ and the sample was tensed up to $35 \%$ at the rate of extension and retraction of $500 \mathrm{~mm} / \mathrm{min}$ according to the standard.

\subsection{Measurement of glove stretch (elastic behaviour)}

The elastic behaviour measurement was carried out through the analysis of the extension of a grid drawn on the gloves when worn on a flexible silicone hand in relaxed and grip postures. A commercially available flexible silicone hand with a circumference of $14.5 \mathrm{~cm}$ was used in this research to simulate the hand of a person wearing glove (Figure 1a). First, a grid of $1 \mathrm{~cm} \mathrm{x} 1 \mathrm{~cm}$ was drawn on a glove in a relaxed posture (Figure 1b). The axial of the grid was numbered. Next, the glove was donned on the flexible silicone hand (Figure 1c). The changes of elastic behaviour of the gloves in a relaxed posture were calculated by measuring the changes in the grid size. The measurements were repeated three times and mean values were reported. After completion of each set of measurements. The glove was doffed off the hand and was allow one-hour relaxation before it was donned for the next consecutive set of measurements. Next, the glove was worn to the flexible silicone hand in a grip posture while holding a plastic ball. The change in the elastic behaviour of the gloves in a grip posture was calculated by measuring the changes in the grid size. Each measurement was repeated three times and the mean values were reported.

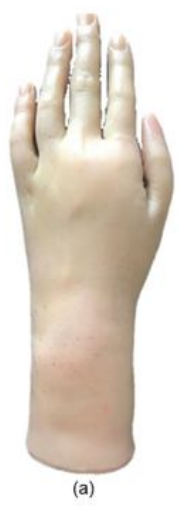

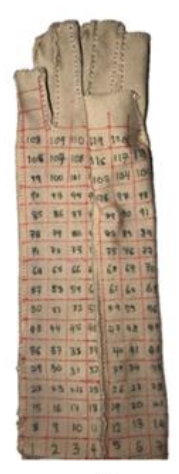

(b)

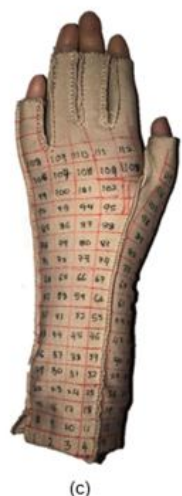

(c)
Figure 1: Measurement of glove stretch (a) flexible silicone hand, (b) grid drawn of relaxed glove, (c) Number grid on flexible silicone hand

In order to show the different level of elastic behaviour undergone by the glove in a relaxed and grip postures, the stretch values were divided into five ranges (Figure 2). The range size was defined as per the formula below (Equation 1).

$$
R=\frac{S m a \text { 尠-Smin }}{N}
$$


Where:

$R$ is the range size

Smax is the maximum stretch, $\mathrm{cm}$

Smin is the minimum stretch, cm

$N$ is the number of ranges $(\mathrm{N}=5)$

\subsection{Mapping and visual representation of glove stretch (elastic behaviour)}

For visual representation of the glove stretch, a different color was allocated to each range (Figure 2). White was used for the minimum range, followed by yellow, gold, orange and red for the maximum range. Each of the grid was colored according to the distribution of the colors in using Microsoft Excel spreadsheet. Both relaxed and grip positions.

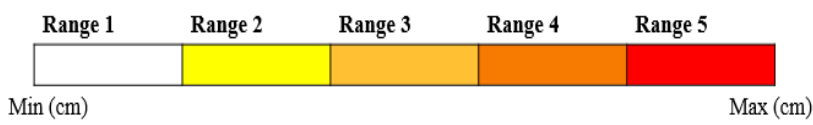

Figure 2: Range of color distributions from minimum to maximum

A 2D map of the glove containing stretch areas was developed by drawing in Adobe Illustrator CS5. The drawing was then superimposed to an image of the flexible silicone hand with the glove donned on. The colored and numbered spreadsheet that contained the data collected was placed under the drawing and was aligned. Once the spreadsheet was aligned with the location of the donned glove on the flexible silicone hand, the ranges were drawn with the pencil tool, using the grid results as a guide (Figure 3 ).
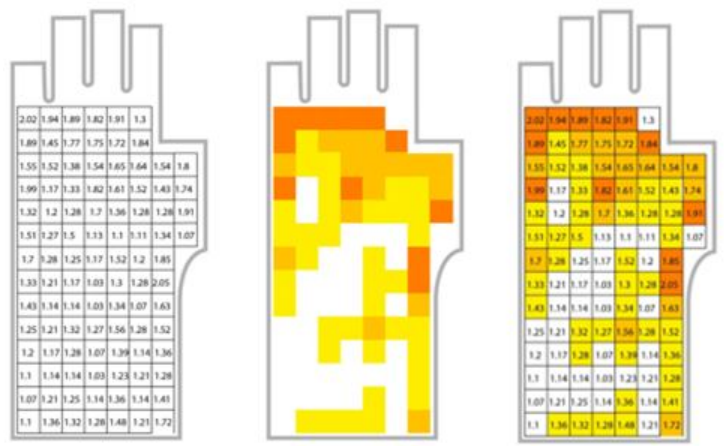

Figure 3: Method for 2D map development

\section{RESULTS AND DISCUSSIONS}

\subsection{Structural and physical attributes of the glove}

The mean mass per unit area for glove $\mathrm{G} 1$ is $285 \mathrm{~g} / \mathrm{m}^{2}$, and for Glove $\mathrm{G} 2$ is $312 \mathrm{~g} / \mathrm{m}^{2}$. The thickness of both gloves is 0.62 $\mathrm{mm}$ for $\mathrm{G} 1$ and $0.78 \mathrm{~mm}$ for $\mathrm{G} 2$. The mean stitch density for glove G1 is 2840 per inch $^{2}$ and Glove G2 3762 per inch $^{2}$.

\subsection{Tensile properties}

Fabrics G1 demonstrated 'square' stretch (also known as 'balanced' stretch) in terms of their tensile performance in both directions (1.9 N/50 mm wale and $2.3 \mathrm{~N} / 50 \mathrm{~mm}$ course). Fabric G2 recorded stress of $3.5 \mathrm{~N} / 50 \mathrm{~mm}$ in wale and 1.7 $\mathrm{N} / 50 \mathrm{~mm}$ in course. The tensile results showed that glove G2 recorded higher stress in wale direction. The stress-strain behaviour of fabrics in the lengthwise (wale) direction affects the bending motion of the hand and finger when the glove is worn, and the stress-strain behaviour in the widthwise direction of the glove affects the magnitude of glove-skin interfacial pressure For a fabric with a higher stress, a lower reduction factor is required, as higher tensile force requires the user to exert greater force to move the hands and fingers [3]. Thus, it is important to select a suitable reduction factor in order to preserve the range of motion of the user.

\subsection{Hand mapping and visual representation of glove stretch (elastic behaviour)}

Figure 4 and Figure 5 shows the mean distribution data of glove stretch collected from relaxed and grip postures for glove G1 and glove G2 when colored according to the range of scale. The colored data based on the range allows one to see the distribution of the glove stretch on the total area of the glove. The vertical side of the scale is the wale direction, while the horizontal side is the course direction. The highest stretch of $2.05 \mathrm{~cm}$ and $2.28 \mathrm{~cm}$ was recorded in glove $\mathrm{G} 1$ and $\mathrm{G} 2$, respectively. The difference in color range distributions demonstrates that a higher range of stretch was recorded in grip posture compared to relaxed posture. This is because when the hand transition from relaxed posture to grip postures, the bones in the metacarpal region of the hand becoming more significant, increasing skin deformation.

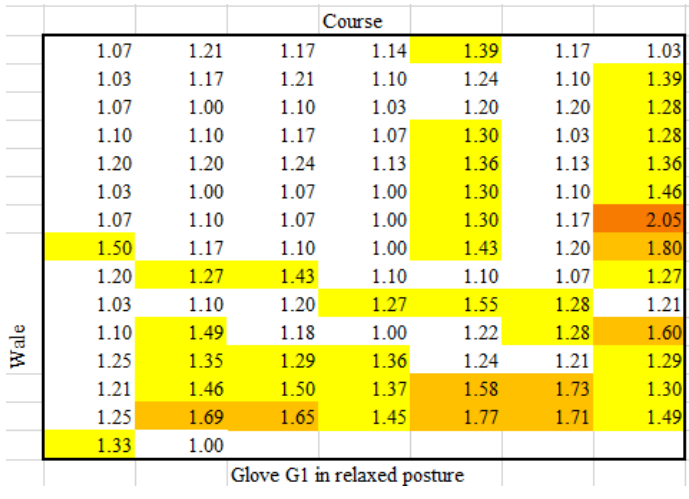

\begin{tabular}{|c|c|c|c|c|c|c|c|}
\hline & & & & & & & \\
\hline & 1.10 & 1.36 & 1.32 & 1.28 & 1.48 & 1.21 & 1.72 \\
\hline & 1.07 & 1.21 & 1.25 & 1.14 & 1.36 & 1.14 & 1.41 \\
\hline & 1.10 & 1.14 & 1.14 & 1.03 & 1.23 & 1.21 & 1.28 \\
\hline & 1.20 & 1.17 & 1.28 & 1.07 & 1.39 & 1.14 & 1.36 \\
\hline & 1.25 & 1.21 & 1.32 & 1.27 & 1.56 & 1.28 & 1.52 \\
\hline & 1.43 & 1.14 & 1.14 & 1.03 & 1.34 & 1.07 & 1.63 \\
\hline & 1.33 & 1.21 & 1.17 & 1.03 & 1.30 & 1.28 & 2.05 \\
\hline & 1.70 & 1.28 & 1.25 & 1.17 & 1.52 & 1.21 & 1.85 \\
\hline & 1.51 & 1.27 & 1.50 & 1.13 & 1.10 & 1.10 & 1.34 \\
\hline & 1.07 & 1.32 & 1.20 & 1.28 & 1.70 & 1.36 & 1.28 \\
\hline & 1.28 & 1.91 & 1.99 & 1.17 & 1.33 & 1.82 & 1.61 \\
\hline & 1.52 & 1.43 & 1.74 & 1.55 & 1.52 & 1.38 & 1.54 \\
\hline & 1.65 & 1.64 & 1.54 & 1.80 & 1.89 & 1.45 & 1.77 \\
\hline & 1.75 & 1.72 & 1.84 & 2.02 & 1.94 & 1.89 & 1.82 \\
\hline & 1.91 & 1.30 & & & & & \\
\hline & & & e G1 i & post & & & \\
\hline
\end{tabular}

Figure 4: Distribution of glove stretch in for glove G1 in different postures 


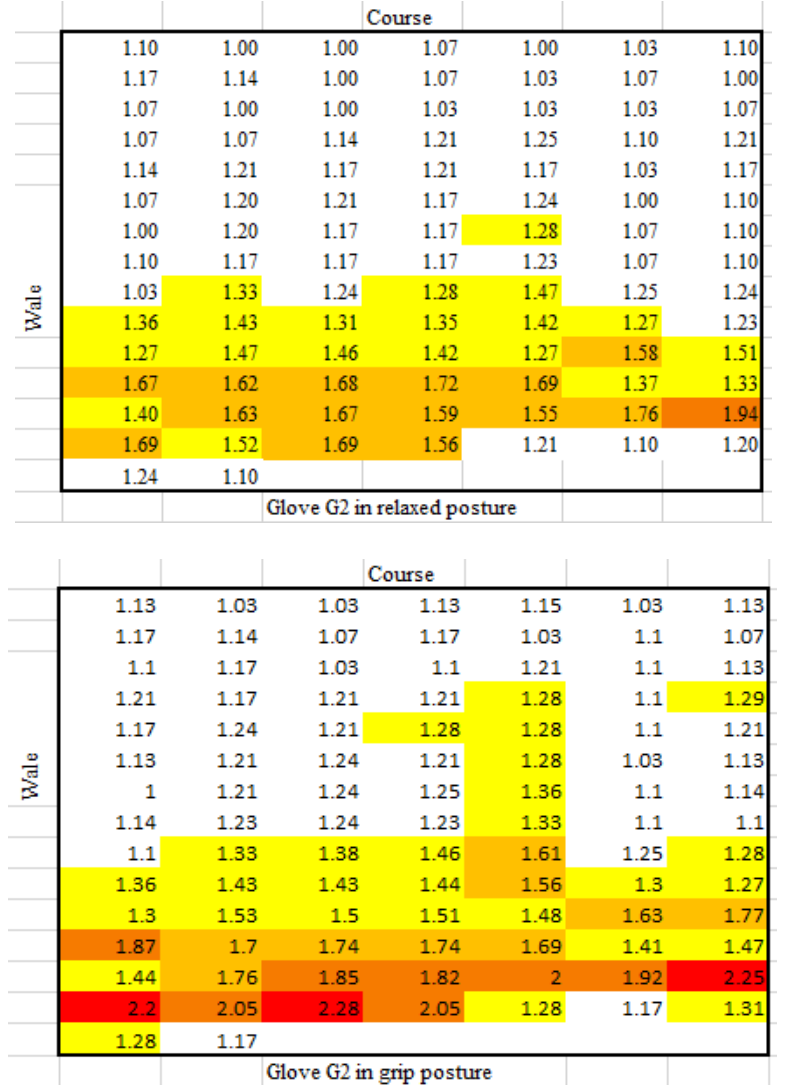

Figure 5: Distribution of glove stretch in for glove G2 in different postures

Figure 6 and Figure 7 demonstrate the frequency of the range of stretch for glove G1 and G2. In the low range, relaxed posture recorded higher frequency compared to grip posture. However, the trend changed in grip posture where the glove stretch was higher and fall into medium to high range.

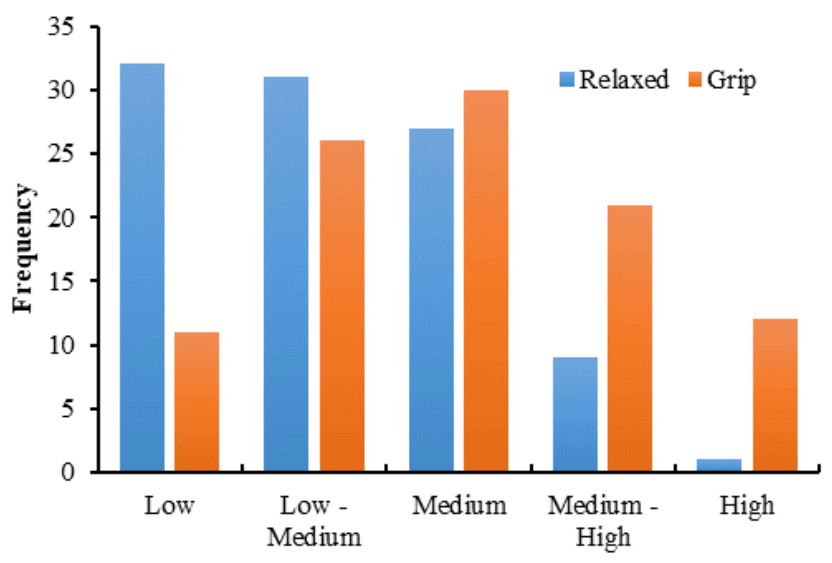

Range of stretch

Figure 6: Range of stretch for glove G1

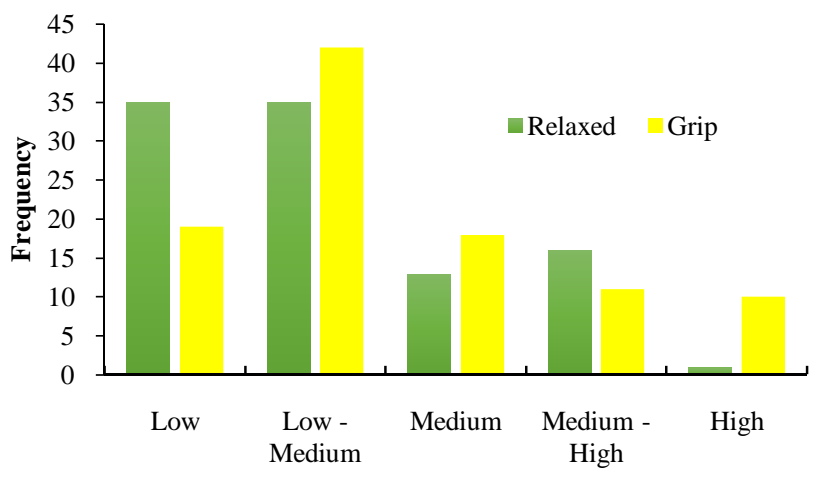

Range of stretch

Figure 7: Range of stretch for glove G1

The maps of the ranges of the stretch were developed for each posture and glove (Figure 8). These maps show the distribution of the stretch areas on the hand with the ranges of higher stretch located on the metacarpal region of the hand. The minimum stretch located around the lower part of the metacarpal-carpal area (near to the wrist). The stretch data revealed that the metacarpal region could potentially make the wearer uncomfortable and restricting the movement of the hand if the glove materials are made of low stretch materials. A lower reduction factor is required for a fabric with higher stress, as a higher tensile force requires the user to exert greater force to move the hands and fingers.

The mapping of the stretch area distributions can provide reliable information on the elastic behaviour of stretchable garment or glove when placed on a body area and the location of the different ranges of stretch for the design and engineering process of functional and comfortable pressure therapy gloves and other user-centric therapeutic garments.

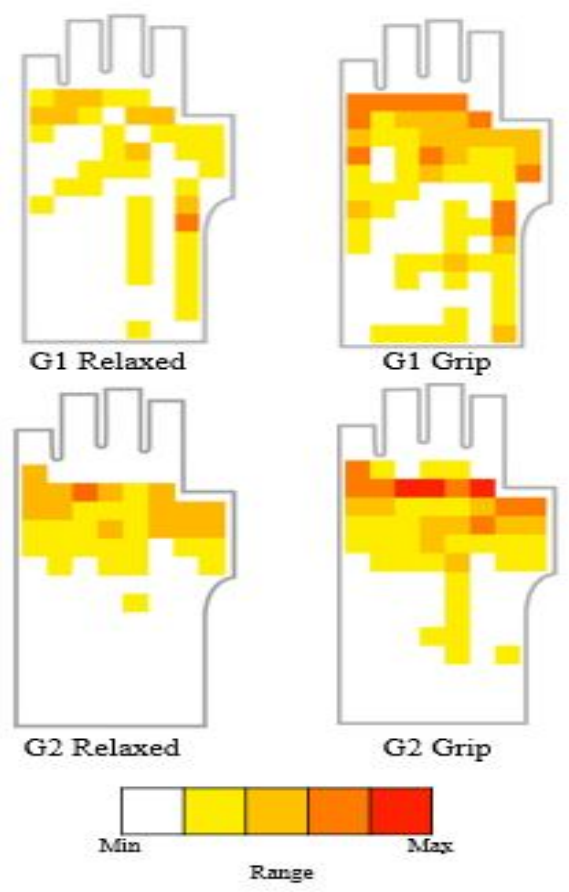

Figure 8: Maps of elastic behaviour range 


\section{CONCLUSION}

Pressure therapy gloves are commonly made of elastic fabrics and engineered with a negative fit to generate pressure on the underlying tissue, compressing and keeping the hand in correct anatomical positions, reducing muscle oscillation and supporting the underlying tissue. The objectives of this study were to analyze the elastic behaviour of pressure therapy gloves to the hand shape and its relation to fabric stretch, as to define the design characteristics required for engineering and developing a functional and comfortable pressure therapy glove. The results of the current study showed that the glove stretches up to $2.28 \mathrm{~cm}(56.14 \%)$. An increase of stretch is assumed to increase the interface pressure of the underlying body part [3], [13], [14]. The glove stretch maps showed the maximum ranges of fabric stretch located around the metacarpal region and increased with the change of posture. When the hand transitions from a relaxed posture to a grip posture, the curvature of the bones in the metacarpal region becomes more significant, which may mean that unnecessary pressure is delivered to this area, making the wearer uncomfortable and potentially restricting the hand movement. The mapping develops in this study can be used for understanding the deformation of the glove during practical wear and determination of ideal reduction factor for developing comfortable and functional pressure therapy gloves. Biomechanical considerations such as convexities and concavities are important to design a garment that is comfortable, allow ease of movement and fulfil its functional purpose.

\section{ACKNOWLEDGEMENT}

The authors would like to thank the Ministry of Education Malaysia for supporting this research under Fundamental Research Grant Scheme Vot No. FRGS/1/2018/TK05/ UTHM/03/4 and partially sponsored by Universiti Tun Hussein Onn Malaysia.

\section{REFERENCES}

1. D. Bagchi, H. Moriyama, and S. P. Raychaudhuri, Arthritis, Aging Society, Exercise, Nutrition, and Other Precautionary Measures, in Arthritis, CRC Press, 2016, pp. 581-584.

2. Manno, R. (2012). Osteoarthritis: Epidemiology \& risk factors. Retrieved from http://www.hopkinsarthritis.org/arthritis-info/osteoar thritis/oa-clinical-presentation/

3. S. Nasir, Therapeutic gloves for patients with arthritis, Ph.D. dissertation, School of Fashion and Textiles, RMIT Univ., Melbourne, Australia, 2016.

4. A. Yu, K. L. Yick, S. P. Ng, and J. Yip, Prediction of fabric tension and pressure decay for the development of pressure therapy gloves, Textile research journal, vol. 83, no. 3, pp. 269-287, 2013.

5. A. Hammond, V. Jones, and Y. Prior, The effects of compression gloves on hand symptoms and hand function in rheumatoid arthritis and hand osteoarthritis: a systematic review, Clinical Rehabilitation, vol. 30, no. 3, pp. 213-224, 2016.

6. N. Sau-Fun, H. Chi-Leung, and W. Lai-Fan, Development of medical garments and apparel for the elderly and the disabled, Textile Progress, vol. 43, no. 4, pp. 235-285, 2011.

7. G. Krimmel, The construction and classification of compression garments, Template for Practice: Compression hosiery in upper body lymphoedema, pp. 2-5, 2009.

8. E. Eltahan, Effect of Lycra percentages and loop length on the physical and mechanical properties of single jersey knitted fabrics, Journal of Composites, vol. 2016, 2016.

9. D. B. Sitotaw and B. F. Adamu, Tensile properties of single jersey and $1 \times 1$ rib knitted fabrics made from $100 \%$ cotton and cotton/lycra yarns, Journal of Engineering, vol. 2017, 2017.

10. J. McCann, Environmentally conscious fabric selection in sportswear design, in Textiles for sportswear, Elsevier, 2015, pp. 17-52.

11. O. Troynikov and C. Watson, Knitting technology for seamless sportswear, in Textiles for sportswear, Elsevier, 2015, pp. 95-117.

12. C. J. Smith and G. Havenith, Body mapping of sweating patterns in male athletes in mild exercise-induced hyperthermia, European journal of applied physiology, vol. 111, no. 7, pp. 1391-1404, 2011.

13. S. H. Nasir, O. Troynikov, and N. Massy-Westropp, Arthritis patients' experience and perception of therapeutic gloves, International Journal of Fashion Design, Technology and Education, vol. 11, no. 2, pp. 233-242, 2018.

14. L. Macintyre and M. Baird, Pressure garments for use in the treatment of hypertrophic scars-a review of the problems associated with their use, Burns, vol. 32, no. 1, pp. 10-15, 2006.

15. O. Troynikov, E. Ashayeri, M. Burton, A. Subic, F. Alam, and S. Marteau, Factors influencing the effectiveness of compression garments used in sports, Procedia Engineering, vol. 2, no. 2, pp. 2823-2829, 2010. 